\title{
Streams and patterns in behavior as challenges for future technologies
}

\author{
John C. FenTress \\ Dalhousie University, Halifax, Nova Scotia, Canada
}

\begin{abstract}
How we divide and recombine processes that underlie integrated patterns of behavior represents one of the most fundamental problems in all behavioral science. Avenues of insight range from naturalistic observations to the most advanced technological advances in the recording and analysis of behavioral sequences. Members of the symposium on "Streams and Patterns in Behavior," Maastricht Conference on Measuring Behavior, offered important complementary approaches to issues that are common in all analyses of behavioral patterns, across time scales and levels of organization. Rodent grooming was emphasized as one model system, but contributors also offered insights on a range of issues relevant to our deeper understanding of exploration, emotions, consciousness, and human neurological disorders.
\end{abstract}

The Measuring Behavior 2008 conference opened with a keynote lecture by Sir Patrick Bateson on techniques for measuring behavior. His emphasis was on the appropriate use of statistical methods to provide quantification of essential issues in behavioral organization (see Martin \& Bateson, 2007). This keynote presentation was followed by a symposium on the analysis of streams and patterns in behavior, organized by J. Fentress (Spink et al., 2008). The two offerings had been coordinated prior to the meeting. In this short article, I shall summarize the symposium highlights, for they set the stage for many of the contributions that followed. I am pleased that this issue of Behavior Research Methods is devoted to the conference made possible by Noldus, for it provided an unusually fruitful combination of technological and scientific advances with respect to behavioral research.

All studies of behavior start with observer perceptions, whatever technology is involved in these perceptions (Figure 1). The first overriding problem is that we are victims of selective attention. We attend to some events at the expense of others, often without a clear rationale for doing so (see Hinde, 1970). Investigators must face the

\section{Starting With Observer Perceptions}

- Selective Attention (cf. Hinde, Abstraction)

- Cognitive Biases

- "Objectivity": Value of Multiple Perspectives

- Key Issue: Internal/External Relations Inevitable $\rightarrow$ Dynamic Relations

Figure 1. fact that all investigators relate to their subjects a foundation of cognitive biases. Definitions of objectivity are often restrained by the methods chosen to measure behavioral events (Fentress, 2008). Simple votes of agreement among investigators who use similar methods are not sufficient. Thus, it is often useful to explicitly utilize different carefully defined perspectives and then see how the results from these perspectives fit together. Finally, whatever behavioral systems we isolate for study are the product of internal processes within these systems and variable responses to broader external surrounds.

A key issue in all studies of behavior is how we divide the stream of events into patterns for analysis. Three issues arise immediately. First, how continuous versus discrete or modular are the various facets of behavior that we observe? Second, how stable versus variable are the patterns we abstract from these behavioral streams? Third, how do we link these patterns across levels and time frames of organization? No one of these questions has a clear answer at the present time. In terms of modularity, it is clear that individual properties of behavior have separable domains of control, but two essential issues follow. (1) There are many ways to divide behavioral streams, just as there are many ways to cut a pie. Different cuts give different answers. This is the problem of behavioral taxonomy, a problem that needs much further investigation. (2) Once the divisions are made, it is critical to look for finer subdivisions along with the roles of broader contextual influences in expression and control. In short, mechanistic and systems perspectives must be examined together. Modern technological advances have begun to make this possible. Two fundamental considerations, repeated frequently during the opening symposium and elsewhere in the conference, follow. 
1. The dynamic-stability continuum in behavior is critical and often difficult to evaluate in a critical, as well as comprehensive, way. Behavior is, by definition, dynamic in its basic ordering properties, but these properties are also anchored within structures that give them a recognizable stability. Otherwise, there would be no definable order, such as what makes Animal or Person A predictably different from Animal or Person B. The issue gets more complex when we recognize that both $\mathrm{A}$ and $\mathrm{B}$ have changing rules and changing stable structures, over time and as a function of contexts of expression. This demands the collection of large databases, which are then subjected to quantitative analysis.

2. Awareness of the levels and time frames of analysis are critical for any generalizations we make from particular windows of evaluation. In the study of human behavior, for example, investigators may move from rules of coordination of broad patterns of expression across individuals to rules of coordination (organization) within these individuals. It is not always easy to cross this divide in a coherent manner. New technological tools can be of enormous help. With respect to time frames, it is obvious that windows of investigation can cross spans from milliseconds to entire developmental trajectories. How do we hook these time frames together, in both their stable and changing rules, across levels of organization?

The fundamental question that crosses each of these issues is how we join together the careful, even intuitive, observations of ethologists, psychologists, and their colleagues with the technological tools that bring these observations into a precise analytical framework. To my mind, that was the overriding theme of the Measuring Behavior 2008 conference (see Figure 2).

Subsequent articles in this special issue of Behavior Research Methods will provide more detailed examples than I provide here. My goal in this short introductory article is to provide a coherent framework of the issues that I think apply to essentially every contribution that follows. To do so, I shall highlight six themes that arose from our invitational symposium.

1. One way to summarize the challenges faced by all members of the conference can be labeled the pieces/ relations problem. We necessarily divide any stream of behavioral events into component processes. We then must ask how these component processes fit together across sequences, time, levels of organization, and so forth. An-

\section{Two Critical Items}

- Levels of Organization ("Order and Co-Order") -e.g., Which Level Most Relevant, Most "Simple"?

\section{- Time Frames of Organization}

-e.g., Integration and Development

-Evolution ("ultimate") $\rightarrow$ Function ("consequence")

- Going From Micro to Macro, and Vice Versa

-Which one "wins?"- Neither! (Bidirectionality)

Figure 2. other way to phrase this is to speak of the evaluation of components in contexts. This puts limits on the value of using strict reductionist methods without regard for how mechanisms operate when embedded in their broader systems of operation. Closely linked is the theme of modularity in behavior and its underlying processes. These are conceptually difficult issues, especially when modules are neither homogeneous (indivisible) in their organization nor entirely immune from extraneous influences. A partial modularity view is now common in fields ranging from child development (Karmiloff-Smith, 1992) to the study of the cellular and genetic mechanisms that together contribute to issues in the biological foundations of evolution and development (Jablonka \& Lamb, 2006; Schlosser \& Wagner, 2004).

2. It is the relative and often changing independence among processes that presents major challenges. Beyond this, there is the issue of interdependence, where the properties of A and B mutually influence one another, thus changing future interactions. In a sense, modules become modulated. A well-known case is the phenomenon of coarticulation in human speech, where the properties of a given phoneme can be altered as a function of both preceding and subsequent articulations. A second, less well-known example concerns central pattern generators in invertebrates. Although it is true that small circuits can generate complex patterns of activity even when isolated, these circuits often change their details of operation when reattached to afferents that are normally present (for reviews, see Fentress \& Gadbois, 2001; Ossenkopp, Kavaliers, \& Sanberg, 1996).

3. In both overt behavior and neural circuits, higher order systems often show stability, whereas underlying processes are highly variable. We can, for example, place food reliably into our mouths while moving our heads and bodies during dinnertime conversations. Single-limb movements can be accomplished through a variety of individual patterns of muscular contraction, and so forth. Even in relatively simple invertebrate neural circuits, such as those seen in isolated lobster gastric mill and cardiac ganglion circuits, overall rhythms can be maintained even though individual neurons change their firing patterns.

4. This leads to tractable challenges, whatever behavioral or neural system is under investigation. Within a given level of analysis, it is possible to track where the stabilities and variations occur. Toothbrush bristles may trace consistent patterns of contact with the teeth through a variety of head, forelimb, and body movements. Relative movements of two animals in a social context can be maintained even though, with reference to an outside observer, the movements of the individual animals appear highly variable.

5. However we divide behavior into units or patterns, it is important to realize that superordinate processes may occur that cross over the divisions that we make. A good example is that of prosody in human speech, where themes such as changes in volume or inflection can modulate the details in expression of multiple speech units, otherwise isolated in terms of their lower order properties (Fentress \& Gadbois, 2001). This leads to an increasing complexity in how we evaluate hierarchical models of behavior (Figure 3). 


\section{Seeking Multiple Layers of Organization}

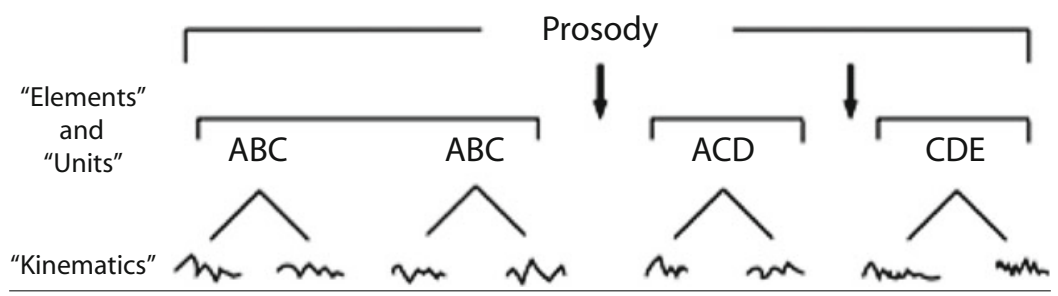

Figure 3.

6. Finally, there is what we mean by hierarchy itself. An issue often raised in this conference concerned what is often called the bottom-up versus top-down approach to behavioral organization. Processes involved in behavior can be viewed most accurately as a dialogue across levels, rather than as simple bottom-up or top-down relations. Clearly, higher order systems are composed through lower order processes. At the same time, these higher order systems can constrain and modulate the very lower order events from which they are composed. Such bidirectional influences are most likely true and, together, provide challenges to our future understanding of behavior, along with the development of new technological tools.

Often the very designation of higher versus lower is a matter of perspective. For example (Figure 4), a given pair of actions (A and $\mathrm{B}$ ) has measurable underlying (subordinately defined) properties. Often, these actions can share certain subordinate properties (designated as " $\mathrm{Y}$ " in the figure), such as when a male lion bites food, its prey, and its mate. We blink in conversations and when stressed, in nonsocial situations, and so forth. This allows, in principle, our views of hierarchical order. "Y" in this context may be considered a superordinate property expressed through Subordinate Actions A and B.

\section{Interim Summary}

A major focus of the present symposium was thus upon the isolation of relatively stable properties of expression that might, from other perspectives, be composed through variable means. It is this linking together of stable and variable properties of behavior that holds particular promise in the development of future technologies. Closely related conceptually is the broader issue of separations and connections in behavior, at whatever level we choose to study it. Abstractions, such as those concerned with inferences about modules, provide a useful starting point (Figure 5).

\section{Rodent Grooming As a Model for Broader Issues}

In his opening address, J. Fentress emphasized the importance of naturalistic observations made from explicitly distinct perspectives. Where are the cohesions and where are the variations in action? How do our measures from different perspectives fit together? To answer such questions, global methods must be combined with reductionist methods. A clear example is the organization of facial grooming in mice (Stilwell \& Fentress, in press).
In a strain-dependent manner, mice utilize a predictable variety of facial grooming and body-oriented strokes that follow one another in sequence. The movements and sequences are not entirely fixed, but with the aid of high-speed photography and computer-based stochastic and time series analyses, probabilistic rules that reflect clear patterns of order can be abstracted. Interestingly, as in the phenomenon of coarticulation in human speech, the details of variation in individual strokes can be accounted for by examining those actions that precede and follow the stroke under investigation. Although the longer strokes and licking movements tend to have a particular time course, there is some variation, which in turn aids the prediction of strokes that will follow. Thus, sequencing and timing must be evaluated together, a task that deserves much more attention.

If letters are used to summarize the strokes, it is then possible to ask how they are assembled into "words," "phrases," and so forth. Just as individual letters can compose different words and the same words can be embed-

\section{Alternative Hierarchies}

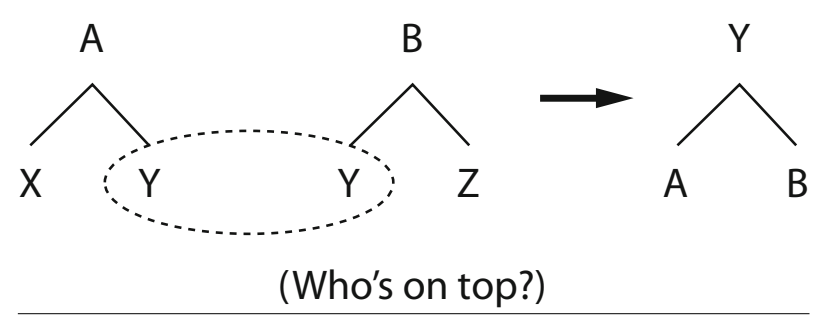

Figure 4.

\section{Separations and Connections}

- Modules: How homogeneous; how separate? \{compartmentalization issue\}

- Independence (separate processes)

- Interactive (plus $\{+\}$ and minus $\{-\}$ )

- Interdependence $(\mathrm{A} \leftarrow \rightarrow \mathrm{B}$ mutually influencing one another) Dynamics among these possibilities

Figure 5. 
ded in different phrases, so too can lower order properties of mouse grooming be shuffled (within definable limits) to form different higher order clusters. Thus, some form of hierarchical analysis is clearly necessary. Interestingly, higher order units can show a consistency even when lower order units show variation, as if the mice occasionally misspell "words" but still elicit clearly recognizable higher order packaging. Although advances in technology clarify these patterning rules, their deeper meanings remain a challenge to the observer.

An additional feature of grooming analysis reflects a simple methodology that can be applied with benefit to the evaluation of nearly all behavioral systems. If, during various aspects of performance, an external stimulus is presented to the mice, their response to the stimulus varies systematically. The simplest example is one in which, during slower and more variable phases of grooming, the ongoing actions are interrupted. During more intense and stereotyped phases of grooming, the mice will frequently ignore the stimulus; that is, the ongoing behavior will continue. This indicates a shift between sensitivity to, even dependence upon, sensory events to a more tightly controlled central patterning. One way to think about this is that, as the behavior becomes more intense and stereotyped in its organization, one can model changes in the focus of underlying mechanisms. There are now a number of studies in the neurobiology of motor control that reflect similar phenomena (Fentress, 1991; Stilwell \& Fentress, in press). In this sense, similar rules can be found across levels of organization, a fact that aids the linking of the levels within a coherent framework.

One other simple assay will be mentioned here. Following facial grooming, mice and other rodents will groom their bellies and then their backs. If a mild stimulus, such as a water drop, is placed on the animals' backs, they will attend to it, but, in most cases, after starting with facial and body grooming, even though there will be increased attention to the back area. The normal sequence is maintained, albeit in a modified fashion in terms of such details as the duration of attention to different body areas. If, however, a stronger stimulus, such as a drop of ether, is placed on the animals' backs, they will attend to it immediately and without the preliminary face and body grooming. Through such simple manipulations, one can thus assay the relative strength or bias of central motor programs.

There are two major lessons from these examples. The first is that it is critical for an observer to document full patterns of behavior if reasonable models are to be obtained. Modern technologies, such as those being developed by Noldus, are a fundamental aid. The second lesson is that even very simple experiments, such as interrupting ongoing patterns of behavior with different classes of stimuli, can help clarify underlying operational principles.

There are more subtle relations among behavioral systems that technologies are now beginning to reveal. For example, it is possible to monitor the relative ongoing strengths of different behavioral tendencies. This indicates that animals are not always "single-minded," in that they have to titrate the relative importance of these tendencies and then make a choice as to which actions to perform. A model we have been working on for some time is that, at moderate intensity, activation of underlying tendencies other than the one an animal is expressing overtly can enhance ongoing performance. At higher levels of activation, these alternative tendencies are most likely to interrupt the same ongoing behavior.

The critical point is that when we evaluate relations among behavioral systems, the relations can vary. The systems can be independent, synergistic, or antagonistic. Which one of these profiles occurs reflects a number of underlying processes, such as timing and the intensity of system activation.

A model with shifting balances of core excitation and inhibitory surround, such as those found in a variety of sensory systems, is promising. As with other models, these ideas can be refined and tested critically only through further advances in technology. In this case, it will be critical to attain more detailed assays of the behavioral tendencies in question.

\section{Symposium Contributions}

J. W. Aldridge and K. C. Berridge. In the symposium, Aldridge and Berridge (2008) summarized aspects of their research showing how specific brain systems contribute distinctively to action sequences, with grooming as a fundamental assay. In their terminology, "rodent grooming has syntactic (rule-driven) sequences with holistic patterns of serial structure" (p. 12), as outlined above. Here, they developed a technology that includes precise evaluations of grooming sequences and recordings from single neurons during different phases of the behavior. Computational properties of participating neural networks can then be derived. The majority of their studies have involved recording from single neurons within various structures of the basal ganglia. Application of dopaminergic drugs and utilization of neurological mutant mice have been combined with these investigations.

Through this rich combination of methodologies, these authors have shown that the neostriatum controls the tightness (stereotypy) of grooming sequences and participates in the processing of sensory stimuli related to broader issues of motivation and emotion. It is now becoming clear that neostriatal mechanisms are also critical in the sequencing of a variety of human actions. Such model systems also connect importantly to a variety of clinical themes in human behavior and neurology, including obsessive-compulsive disorders, Tourette's syndrome, and Parkinson's disease. Thus, technological advances in model systems such as rodent grooming have begun to reveal principles of behavioral organization that have broad significance.

One of the authors' discoveries that deserves special mention is that in hyperdopaminergic mutant mice, grooming patterns fall into a sequential super-stereotypy form of expression. Connected with the knockdown mutation of the dopamine transporter gene (DAT), this superstereotypy in expression produces sequences that are more resistant to interruption. The syntactical chaining of grooming thus tightens along with a shift toward more exclusive patterns of central control. Possible connections 
with human obsessive-compulsive disorders are promising from these and related model systems.

A. Kalueff and J. L. LaPorte. Kalueff and LaPorte (2008) presented a paper that showed that grooming probability and its patterning can be used to assay a number of behavioral stressors and, thus, might connect to affective disorders in humans, such as anxiety, depression, and obsessive-compulsive behavior. They combined the use of environmental stressors, pharmacologically treated animals, and genetically different animals (anxious and nonanxious strains) to model these systems in greater detail. Unfortunately, Kalueff was unable to attend the meeting due to personal conflicting events. Fentress thus gave the presentation he and his colleague had prepared.

Their studies confirm that grooming is often seen in low-stress "comfort" conditions but can also be triggered by stressors, including novelty, predators, water misting, and various drugs and hormones. The supporting data previously mentioned emphasize that only through careful documentation of the sequencing, timing, and intensity of actions such as grooming across levels and contexts of expression will it be possible to refine such model systems.

Using a grooming analysis algorithm, the authors have shown how stress can disorganize grooming, such as through the cephalo-caudal patterning and regional distribution of strokes, incorrect transitions between strokes, and increases in the number of incomplete and interrupted bouts. Thus, global measures of grooming in themselves are not sufficient for more detailed quantitative models. For example, when rats were stressed with exposure to a brightly illuminated novel environment, the time spent grooming was raised, but so were incorrect transitions and the percentage of interrupted bouts. These observations were then combined with pharmacological manipulations previously shown to modulate stress responses. Anxiolytic diazepam was found to lower the percentage of incorrect transitions and interrupted grooming bouts, whereas anxiogenic pentylenetetrazole increased the duration of grooming, with a higher percentage of incorrect transitions and interrupted bouts.

Additional observations with vitamin D receptor knockout mice demonstrated that novelty-induced grooming in these animals was marked by higher percentages of forepaw, head, and hind leg grooming, with less caudal grooming than is seen in wild-type mice. With artificial swiminduced grooming, there were no genotype differences between the groups. The latter observation emphasizes again the importance of examining behavior in multiple contexts if comprehensive pictures are to be obtained.

I. Golani and Y. Benjamini. Golani, who pioneered the use of the Eshkol-Wachmann movement notation system in animal studies, presented a paper with Benjamini in which further problems of behavioral measurement were highlighted (Golani \& Benjamini, 2008). They provided the dissection tool of behavioral genetics (QTL method) in combination with sophisticated computer-based kinematic analyses. These analyses included elaborate smoothing and data segmentation procedures, combined with first, second, and third derivative measures such as velocity, acceleration, jerk, and curvature of ongoing animal actions.

Tracking systems that included both overall path transitions and precise measures of interlimb coordination at the joints scale provided the raw data. They then took derived measures of discrete patterns across levels of organization as the foundation for model systems. Their studies confirmed that the contents of these carefully documented patterns is variable and cautioned that the variable contents of patterns must be included in any analysis. Otherwise, one is left with "black boxes," which are incomplete and can be misleading at the explanatory level. One of the important implications of the authors' research is that early ethological analyses of action sequences (ethograms) have limited value for evaluations of the comparative dynamics that underlie these actions. In their terms, "since the time-series of kinematic data are indexed, segmentation is performed at the indexing level, leaving the kinematic time-series intact and accessible for any other type of analysis or any other type of segmentation" (p. 8). By looking at segmentation in several distinct but compatible ways, a comprehensive picture of action sequences can be obtained.

The richness of this approach can be appreciated by the authors' studies of exploratory behavior, in which the paths taken by mice were evaluated in terms of both lingering (with documentation of "preferred places") and progression episodes on the basis of speed profiles. Distinctions in these measures between strains are highly heritable. As further documentation, mice were evaluated in both familiar and novel environments. In the former, the mice perform vertical as well as horizontal head scans, whereas in novel environments, the scans are restricted to horizontal movements. The importance of dissecting and reconnecting actions across complementary levels of performance thus becomes clear.

Within all such studies, relations between the timing and sequencing of animal and human actions offer many challenges. For example, a given action may follow other actions in predictable sequence or may occur at particular time intervals, independently of intervening actions. In each case, both the stability and variation of action patterns deserve evaluation, a task that, without advances in technology, has proven to be intractable. Put into other terms, if timing affects sequencing and sequencing affects timing, analyses of one or the other in isolation are bound to lead to incomplete evaluations. One can add variables, such as measures of behavior intensity (duration, limb velocity, and the like).

M. Magnusson. In his presentation, Magnusson (2008) reminded us that unaided human perceptions of behavior can be akin to something like trying to decipher a mobile Rorschach projective test. Without precise and objective measures, we cannot move forward in a systematic and critical way. He emphasized mathematics as the search for patterns in nature, with examples that included fractal, chaos, and symmetry/group mathematics. In his terms, "regarding measurement, obviously, phenomena in nature that cannot be detected, cannot be counted, classified, or analyzed in any way" (p. 10). Domain-specific 
analyses are now available that are gradually replacing domain-independent statistical analyses. In this regard, a variety of complex behavioral profiles, such as those seen in human nonverbal interactions, are beginning to be revealed through technological advances. In this as in most other cases, reference of component properties to higher order spatial and temporal envelopes is necessary.

Through the design of specialized algorithms, Magnusson (2008) has been able to isolate both sequential and nonsequential aspects of behavioral pattern formation, along with their spatial distribution. Importantly, with the development of the Theme software, he has opened new avenues for dealing with sequential and temporal aspects of behavioral expressions within a single coherent framework. The methods are abstract, in the sense that they can be applied from levels of order that range from social interactions to interactions among neurons in central nervous system networks.

One of the powerful applications Magnusson (2008) outlined is how his computer methodologies can reveal subtle patterns in human facial expressions. Individual movement properties are then statistically classified into higher order properties of expression, which are then related to the specific contexts within which the actions are elicited and combined. Special analyses, called t-patterns, have revealed a number of initially surprising facts. For example, cyclically occurring t-patterns can be composed of elements that, when isolated, do not share the cyclical occurrence of the higher order t-patterns. The questions then arise as to what are the properties of these individual elements and how their arrangements together allow for higher order cyclical events.

M. and A. J. Cabanac. M. and A. J. Cabanac (2008) offered suggestions from careful comparative studies of how we might, in the future, begin to attain a better understanding of higher order behavioral phenomena, such as consciousness. Concepts such as consciousness are often ambiguous in their definition. The presenters thus suggested that fundamental insights can be obtained through the systematic evaluation of four dimensions: quality, intensity, hedonicity, and duration. The result is complex mental activity (e.g., thoughts, feelings, emotions) that spans time frames that exceed simple stimulus-response models of behavior. Included within this package is a sense of oneself as embedded outside of oneself.

From a comparative perspective, a fundamental question is how consciousness evolved and how and to what extent it is shared among nonhuman species (e.g., Darwin, 1872). The Cabanacs reminded us that, even in human affairs, our inferences of consciousness in others is dependent on the measurements and assumptions we make. Although behavioral measures in this context are obviously indirectly related to inferences of higher order phenomena, such as consciousness, the authors provided data from what they labeled as emotional fever, along with tachycardia and behavioral mimics of sensory pleasure. From these studies, they concluded that at least certain signs of consciousness can be found in mammals, birds, and reptiles, but not in amphibians and fish. It will be exciting to see where future investigations along these and related lines lead.

\section{Conclusions}

For the purposes of this article, the critical point is that, with the help of various forms of technology and measurement, questions are being asked in a much richer, as well as a more precise, way than was previously possible. Clearly, this has much scope for future technological advances, but for these to be maximally effective, it is critical that they be tied to issues in behavior that move beyond technologies in isolation.

N. Tinbergen, the 1973 Nobel Laureate in ethology, once made a good point here. He pointed out that ethology can often be contrasted to other fields of investigation in that ethologists apply simple strategies for observing natural patterns of behavior and then ask what, if any, technological innovations are needed to provide further insights. He contrasted this with other areas of investigation, in which investments are often made in expensive and imperfect technologies, where the question of what aspects of behavior might now be measured then arises. Obviously, it is the convergence of these two perspectives that is most critical, as was emphasized by all of the presenters in the streams and patterns symposium. We look to future advances, along all fronts. Relevance and precision are essential partners, and while we advance the "boxes" of technology, we must also be willing to step outside these "boxes" to isolate problems that deserve further investigation, however difficult that might be at the moment.

Put in basic terms, every action or underlying action process occurs within the context of proceeding, concurrent, or subsequent action tendencies. It is how we may best evaluate both the individual and combined properties of behavior in terms of their sequential, temporal, and hierarchical properties, along with the broader contexts within which these properties are expressed, that represents our most fundamental challenges. Contexts include not only environmental events that are occurring at the moment, but also genetic foundations, along with developmental histories that influence intrinsic patterns of organization plus responses to these events. The order is a tall one, one that technological advances are beginning to help us embrace in precise terms across many levels and time scales of organization.

\section{AUTHOR NOTE}

Correspondence concerning this article should be addressed to J. C. Fentress, Department of Psychology and Neuroscience, Dalhousie University, Halifax, Nova Scotia, Canada (e-mail: fentress@dal.ca).

\section{REFERENCES}

Aldridge, J. W., \& Berridge, K. C. (2008). Brain systems for action sequences. In A. J. Spink et al. (Eds.), Proceedings of Measuring Behavior 2008 (p. 12). Wageningen, The Netherlands: Noldus Information Technology.

Cabanac, M., \& Cabanac, A. J. (2008). Measuring consciousness in animals. In A. J. Spink et al. (Eds.), Proceedings of Measuring Behav- 
ior 2008 (p. 45). Wageningen, The Netherlands: Noldus Information Technology.

DARWIN, C. (1872). The expression of emotions in man and animals. London: Murray.

FENTRESS, J. C. (1991). Analytical ethology and synthetic neuroscience. In P. Bateson (Ed.), The development and integration of behaviour (pp. 77-120). Cambridge: Cambridge University Press.

Fentress, J. C. (2008). Stepping outside the traditional "science" box. In A. J. Spink et al. (Eds.), Proceedings of Measuring Behavior 2008 (p. 5). Wageningen, The Netherlands: Noldus Information Technology.

Fentress, J. C., \& GadBois, S. (2001). The development of action sequences. In E. Blass (Ed.), Handbook of behavioral neurobiology (pp. 393-431). New York: Kluwer Academic/Plenum.

Golani, I., \& Benjamini, Y. (2008). Problems of behavior measurements. In A. J. Spink et al. (Eds.), Proceedings of Measuring Behavior 2008 (p. 8). Wageningen, The Netherlands: Noldus Information Technology.

Hinde, R. A. (1970). Animal behaviour: A synthesis of ethology and comparative psychology (2nd ed.). New York: McGraw-Hill.

JABLONKA, E., \& LAMB, M. J. (2006). Evolution in four dimensions. Cambridge, MA: MIT Press.

KALUefF, A. V., \& LAPORTE, J. L. (2008). Understanding brain affective states by measuring animal grooming patterning. In A. J. Spink et al. (Eds.), Proceedings of Measuring Behavior 2008 (p. 9). Wageningen, The Netherlands: Noldus Information Technology.
Karmiloff-Smith, A. (1992). Beyond modularity: A developmental perspective on cognitive science. Cambridge, MA: MIT Press.

MaGnUsson, M. S. (2008). Observing versus seeing, perception versus detection, and data versus nature. In A. J. Spink et al. (Eds.), Proceedings of Measuring Behavior 2008 (p. 10). Wageningen, The Netherlands: Noldus Information Technology.

Martin, P., \& Bateson, P. (2007). Measuring behavior: An introductory guide. Cambridge: Cambridge University Press.

Ossenkopp, K.-P., Kavaliers, M., \& SAnberg, P. R. (Eds.) (2001). Measuring movement and locomotion: From invertebrates to humans. Austin: Landis.

Schlosser, G., \& Wagner, G. P. (Eds.) (2004). Modularity in development and evolution. Chicago: University of Chicago Press.

Sink, A. J., Ballintijn, M. R., Bogers, N. D., Grieco, F., Loijens, I. W. S., Noldus, L. P. J. J., ET AL. (EDs.) (2008). Proceedings of Measuring Behavior 2008. Wageningen, The Netherlands: Noldus Information Technology.

Stilwell, F., \& Fentress, J. C. (in press). Grooming sequencing and beyond: How it all began. In A. V. Kalueff, J. L. LaPorte, \& C. L. Berner (Eds.), Neurobiology of grooming behavior. Cambridge: Cambridge University Press.

(Manuscript received November 1, 2008; revision accepted for publication January 14, 2009.) 